adverse events during the follow up period. Average patient travel time and distance for follow up was reduced. Hospital transport was not required for any patient. The feedback from patients was positive and based around ease of travel, length of travel and stay, and flexibility to attend.

Conclusions and Implications This pilot study demonstrates the feasibility and benefits of remote pacemaker monitoring from a community location. This model could be replicated in the future by other Trusts.

\section{PATIENTS ADMITTED TO HOSPITAL WITH A DIAGNOSIS OF ATRIAL FIBRILLATION OUTSIDE OF STANDARD WEEKDAY WORKING HOURS AND AT WEEKENDS HAVE WORSE MORTALITY AND POORER SURVIVAL}

${ }^{1}$ Paul R Carter, ${ }^{2}$ Jennifer Reynolds, ${ }^{3}$ Hardeep Uppal, ${ }^{4}$ Suresh Chandran, ${ }^{5}$ Jaydeep Sarma, ${ }^{6}$ Rahul Potluri* ${ }^{1}$ Royal Free London NHS Foundation Trust; ${ }^{2}$ University of Birmingham; ${ }^{3}$ ACALM Study Unit in Collaboration with Aston Medical School; ${ }^{4}$ North Western Deanery; ${ }^{5}$ Deaprtment of Cardiology, University Hospital of South Manchester; ${ }^{6}$ ACALM Study Unit in Collaboration with Aston Medical School, Aston University; *Presenting Author

\subsection{6/heartjnl-2016-309890.60}

Introduction Atrial fibrillation (AF) is the most common cardiac arrhythmia and is associated with serious sequelae such as stroke and a significant mortality rate. Timely and optimal treatment of this condition can minimise these complications. There is evidence that patients with a range of conditions admitted out-of-hours (during evenings and weekends) have worse mortality compared to patient admitted during standard working hours. Mortality rates of AF patients admitted out of hours in the UK have not been previously been studied. We therefore aimed to study the impact of day and time of admission on short and long-term mortality in patients with AF.

Methods Anonymous data was collected from all patients with a diagnosis of AF admitted to several NHS hospitals in the

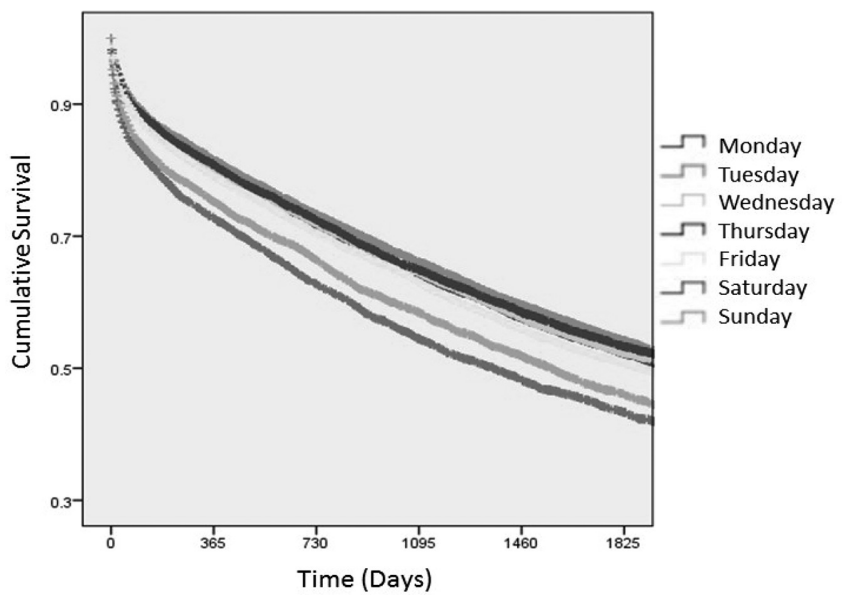

Abstract 60 Figure 1

North of England from $1^{\text {st }}$ January 2000 to $31^{\text {st }}$ March 2013 using the ACALM study protocol. The primary outcome of the study was "in and out of hospital mortality" within 30 days, 1 year, 5 years after the index admission.

Results 929,552 patients were admitted, of which 42,687 (4.6\%) patients had AF. 18,732 (43.9\%) of these were admitted during normal working hours (0900-1700; MondayFriday) and 23,955 (56.1\%) were admitted out of hours. Unadjusted crude mortality rates of patients admitted out of hours $(539 / 1000)$ were higher than those admitted during normal working hours (489/1000). Unadjusted crude death rates of patients admitted on Saturday (588/1000) and Sunday $(548 / 1000)$ were significantly higher than other days of the week (Figure 1). Cox regression analyses accounting for variations in age, gender, ethnic group and the ten most common causes of death in the UK (Listed in Table) showed that out of hours admission with AF conferred significantly worse

Abstract 60 Table 1 Demographics and comorbidities of atrial fibrillation patients

\begin{tabular}{|c|c|c|c|}
\hline & All patients & Admitted during normal hours $(0900-1700$ Monday to Friday) & Admitted outside of normal working hours \\
\hline Age, years $\pm S$. D & $74.2 \pm 12.5$ & $73.8 \pm 12.0$ & $74.5 \pm 12.9$ \\
\hline Male & $21891(51.3 \%)$ & $9881(52.7 \%)$ & $12010(50.1 \%)$ \\
\hline Female & $20796(48.7 \%)$ & $8851(47.3 \%)$ & $11054(49.9 \%)$ \\
\hline Ethnicity & $38052(89.1 \%)$ & $16900(90.2 \%)$ & $21152(88.3 \%)$ \\
\hline Caucasian & $735(1.7 \%)$ & $280(1.5 \%)$ & $455(1.9 \%)$ \\
\hline South Asian & $283(0.7 \%)$ & $118(0.6 \%)$ & $165(0.7 \%)$ \\
\hline Afro Caribbean & $107(0.3 \%)$ & $44(0.5 \%)$ & $63(0.3 \%)$ \\
\hline Oriental & $51(0.1 \%)$ & $21(0.1 \%)$ & $30(0.1 \%)$ \\
\hline Mixed & $3459(7.2 \%)$ & $1369(7.1 \%)$ & $2090(8.7 \%)$ \\
\hline \multicolumn{4}{|l|}{ Other } \\
\hline IHD & $13900(32.6 \%)$ & $6214(33.1 \%)$ & $7686(32.1 \%)$ \\
\hline Heart Failure & $10992(25.8 \%)$ & 4599 (24.6\%) & $6393(26.7 \%)$ \\
\hline Lung Cancer & $926(2.2 \%)$ & $431(2.3 \%)$ & $495(2.1 \%)$ \\
\hline Breast Cancer & $810(1.9 \%)$ & $356(1.9 \%)$ & $454(1.9 \%)$ \\
\hline Colon Cancer & $340(0.8 \%)$ & $170(0.9 \%)$ & $170(0.7 \%)$ \\
\hline COPD & $6379(14.9 \%)$ & 2741 (14.6\%) & $3638(15.2 \%)$ \\
\hline Pneumonia & $5414(12.7 \%)$ & $2101(11.2 \%)$ & $3313(13.8 \%)$ \\
\hline CVD & $5007(11.7 \%)$ & $1934(10.3 \%)$ & $3073(12.8 \%)$ \\
\hline CKD & $4062(9.5 \%)$ & $1721(9.2 \%)$ & $2341(9.8 \%)$ \\
\hline Dementia & $3570(8.4 \%)$ & $1309(7.0 \%)$ & $2261(9.4 \%)$ \\
\hline
\end{tabular}

$\mathrm{IHD}=$ Ischaemic heart disease; $\mathrm{COPD}=$ Chronic Obstructive Pulmonary Disease; $C K D=$ Chronic Kidney Disease; CVD= Cerebrovascular Disease 
mortality (OR 1.12 (95\%C. I 1.09-1.15) compared to admission during normal working hours.

Conclusions This study suggests a higher risk of death for patients with a diagnoses of Atrial Fibrillation admitted outside of normal hours and weekends compared with standard weekday normal working hours. The impact of our findings on service provision and healthcare delivery should to be widely debated.

\section{ANTICOAGULATION FOR ATRIAL FIBILLATION IN THE ELDERLY}

Sarah Beth McClelland, Victoria Wilson*, Niall Hughes. NHS Greater Glasgow and Clyde; *Presenting Author

\subsection{6/heartjnl-2016-309890.61}

Introduction The incidence of atrial fibrillation (AF) increases with age and carries with it a 5 fold increased risk of having a stroke. The most effective prevention is anticoagulation yet elderly people are often viewed of as high risk and are not started on therapy. The first cycle of this audit included 166 current inpatients of the Care of the Elderly Department (COTE) in Gartnavel General hospital (GGH) aged 65 and over. Over one third (36\%) of admissions had AF however only $33 \%$ of these were on anticoagulation. 11/40 (28\%) of those with AF had no decision about anticoagulation recorded. All patients had a CHADSVASC score of 2 or greater so should have been considered for anticoagulation. Novel anticoagulation (NOACs) are now available as first line treatment of non-valvular atrial fibrillation accounting for $50 \%$ of those anticoagulated with the remaining $50 \%$ on Warfarin.

Intervention The audit findings were presented to the GGH COTE department and included the current guidelines for AF and use of NOACs. A copy of the presentation was also sent to all departmental staff via email. Information is now included in the junior doctor departmental handbook given at induction, and a section in the admission document regarding Atrial Fibrillation must be completed for each patient.
Results A regular audit of the COTE department in the form of Plan Do Study Act (PDSA) cycles was implemented to monitor the effectiveness. In March 2015 the first cycle after initial intervention, $60 \%$ of those with $\mathrm{AF}$ were receiving anticoagulation, however during April (junior doctor changeover) this fell to $23 \%$. Further education was implemented and $50 \%$ of patients were on anticoagulation in May. There was also increasing compliance with filling in the admissions box regarding $\mathrm{AF}$, rising from 39\% at baseline to $71 \%$ in May.

Discussion All new admissions to COTE departments should be assessed for atrial fibrillation and considered for anticoagulation including use of NOACs as an alternative to warfarin. Having a section regarding $\mathrm{AF}$ in the admissions booklet is a useful prompt for discussion of anticoagulation.

\section{LEFT ATRIAL APPENDAGE OCCLUSION FOR STROKE PREVENTION IN ATRIAL FIBRILLATION: CONTEMPORARY EXPERIENCE FROM A COMMISSIONING THROUGH EVALUATION SITE}

${ }^{1}$ Stefano Bartoletti ${ }^{*}$, ${ }^{1}$ Periaswamy Velavan, ${ }^{2}$ Jennifer Barclay, ${ }^{1}$ Lindsay Morrison, ${ }^{1}$ Afshin Khalatbari, ${ }^{3}$ Timothy Fairbairn, ${ }^{4}$ Nikhil Sharma, ${ }^{1}$ Dhiraj Gupta. 'Liverpool Heart and Chest Hospital NHS Foundation Trust; ${ }^{2}$ University of Liverpool School of Medicine; ${ }^{3}$ Liverpool Heart and Chest Hospital NHS Trust; ${ }^{4}$ The Royal Liverpool and Broadgreen University Hospitals NHS Trust; *Presenting Author

\subsection{6/heartjnl-2016-309890.62}

Background Since October 2014, NHS England has approved funding for left atrial appendage occlusion (LAAO) for stroke prevention in patients with atrial fibrillation in $10 \mathrm{UK}$ sites as part of Commissioning through Evaluation (CtE) process. There are no data available on contemporary LAAO practice in the CtE era.

Methods In July 2014, we instituted several processes to ensure compliance with stringent $\mathrm{CtE}$ requirements. These included creation of a multidisciplinary team (MDT) that included stroke physicians and non-invasive cardiologists with interest in cardiac imaging, agreement on objective inclusion

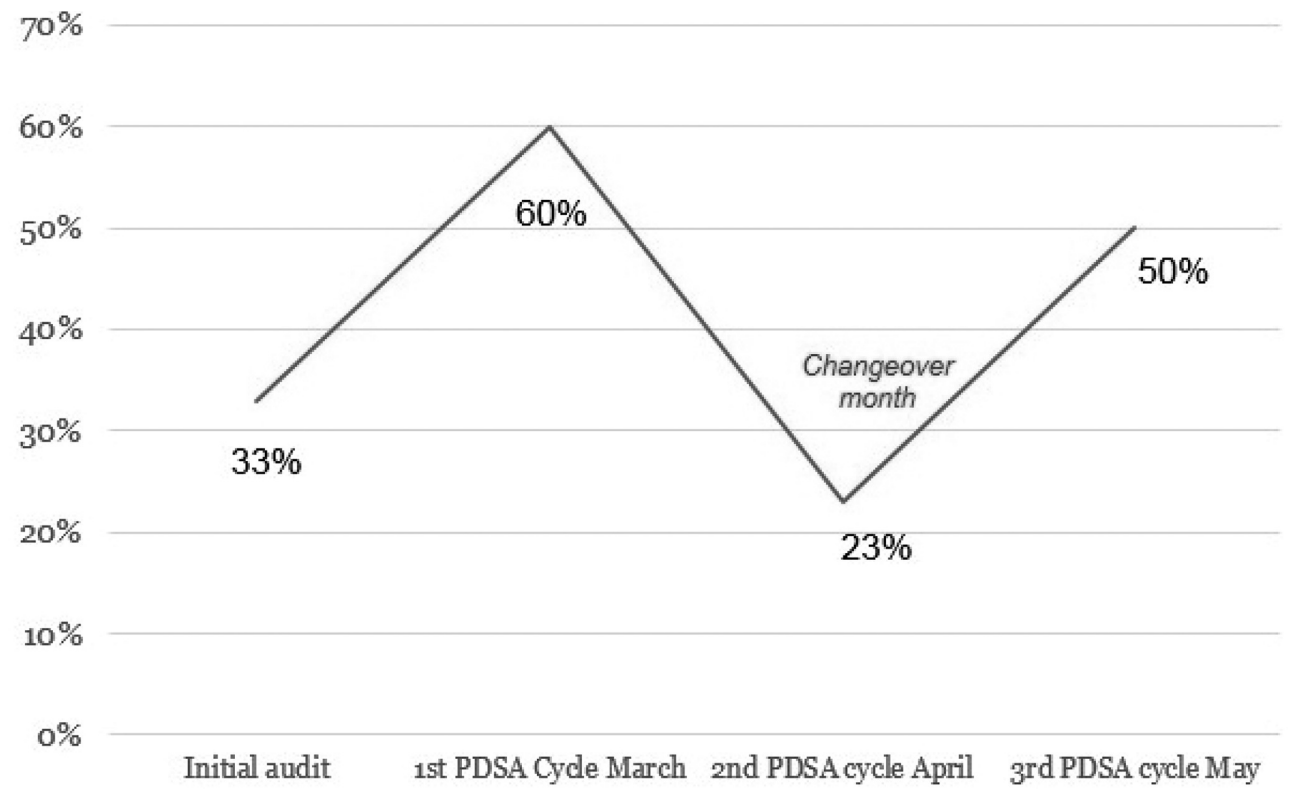

